Proc. Indian Acad. Sci. (Earth Planet. Sci.), Vol. 95, No. 2, July 1986, pp. 223-236.

(C) Printed in India.

\title{
Inverse problem for the determination of the bounds on the mantle heat flow
}

\author{
R N SINGH ${ }^{\dagger}$ and G VASSEUR* \\ ${ }^{\dagger}$ National Geophysical Research Institute, Hyderabad 500007, India \\ *Centre Geologique et Geophysique, USTL, 34060 Montpellier Cedex, France.
}

\begin{abstract}
This paper presents a method for determining the nature of the spatial structure of the heat flow anomaly at depth given a few observations of surface heat flow. We use the constraint of the positivity of the heat flow throughout the domain under consideration to arrive at the solution. Both Parker's ideal body and linear programming approaches have been considered. We find that a few measurements are able to give a reasonable good estimation of the heat flow anomaly at depth. The method has been applied to a real case in the French Massif Central. Results point to the presence of enhanced basal heat flow anomaly.
\end{abstract}

Keywords. Inverse problem; linear programming; mantle heat flow.

\section{Introduction}

Heat flow measurements and their interpretations are of primary importance for the knowledge of earth's internal state and processes. In last few decades numerous heat flow measurements have been made in various tectonic provinces of both continental and oceanic regions. These observations have led to the widely accepted idea that below the lithosphere heat is brought up as a result of (global and local) convective systems and that, within the lithosphere and in particular in the crust, this heat, supplemented by heat due to radioactive decay, is brought to the surface by conductive processes (Sclater et al 1980). In the continental area, where the average heat flow is about $65 \mathrm{mWm}^{-2}$, there exist numerous areas where the observed heat flow values are anomalous showing high values and short space scales. For instance, a recent SinoFrench expedition in Southern Tibet obtained a high heat flow value of $146 \mathrm{mWm}^{-2}$, within a short distance of $25 \mathrm{~km}$ (Francheteau et al 1984), reminiscent of similar observations in trench areas (Blackwell et al 1982). These anomalies can be explained either by enhanced radiogenic or magmatic heat in the crust or/and enhanced regions of heat flow/temperature below the crust. It is crucial to derive the nature of these perturbing processes from the few available heat flow data as they are responsible for a variety of geological, geochemical and geophysical phenomena. These problems involving inferences from the finite number of noisy data are ideally suited for the applications of inverse methodologies developed in the recent literature.

Geophysical inverse problems have a long history but their resolutions proliferated after the development of the Backus-Gilbert method (Backus and Gilbert 1967, 1968, 1970) and its various simplifications/generalizations/sophistications (Backus 1972; Tarantola and Valette 1982; Oldenburg 1983; Jackson and Matsu'ura 1985; Spensor 1985). Parker (1977 a,b) has provided a lucid review of these problems and techniques. The problems here essentially reduce to determining the distribution of the physical properties (represented by piecewise continuous functions) from a finite set of data 
(which contain noises), a non-unique proposition. Such problems could be stable or unstable, in the sense that a small error in the initial/boundary data would lead to unacceptable large values of the fields. These problems have been sought to be resolved by prescribing a class of models which are constrained by the data and by showing how the input of additional data would further constrain the model. Often, the solutions are given with associated resolution widths, the bounds on the model parameters are prescribed, or unique properties are chosen which are common to all solutions.

Parker $(1974,1975)$ has pioneered an ideal body solution approach to geophysical inverse problems. This approach seeks, in general, the lower bound on the maximum absolute value of piecewise-continuous functions representing the class of all solutions. Given this solution, it can be confidently asserted that the true solution would reach or exceed this value somewhere in the region under consideration. Huestis (1982) has given a geometrical interpretation of the Parker's ideal body solution by reducing the infinite-dimensional problem to a finite-dimensional one and using the Fenchel Duality Theorem. For few data cases the solution can be obtained analytically or graphically. However for a large data set, recourse has to be made to linear programming (LP)/methods pioneered by Safon et al (1977) following the formulation of Sabatier (1977 a,b), Huestis and Parker (1977), Huestis (1979) and Garmany et al (1979). If we are seeking an answer to the question of existence of the high heat flow region at depth, such an approach would be highly useful. Our work presented here is directed towards this goal. Some of the recent LP applications are by Bayer and Lesquer (1978), Bayer and Cuer (1981), Grasso et al (1983) and Richards et al (1984).

The problem of obtaining the ideal body solution i.e. minimising $\alpha$,

$$
\alpha=\max |x(t)|,
$$

given the constraints connecting $y$ data with $x$ parameters (to be determined) as

$$
\underset{\mathrm{M} \times \mathrm{N}}{\boldsymbol{A} \times \mathrm{N} \times \mathrm{l}}=\underset{\mathrm{M} \times \mathrm{I}}{\mathrm{y}}
$$

(or more generally

$$
\mathbf{y}-\delta \leqslant A \mathbf{x} \leqslant \mathbf{y}+\delta
$$

where $\delta$ is error) and

$$
\mathbf{x} \geqslant 0 \text {, }
$$

can be converted into a linear programming problem using $(N+1)$ slack variables $\left(\tau_{n}, \alpha\right)$ (Sabatier $1977 \mathrm{a}, \mathrm{b}$; Safon et al 1977) as

$$
\begin{aligned}
& \sum_{j=1}^{N} A_{i j} x_{j}=y_{i} \quad(i=1,2, \ldots, M), \\
& x_{n}+\tau_{n}-\alpha=0 \quad(n=1,2, \ldots, N), \\
& x_{n} \geqslant 0 \\
& \tau_{n} \geqslant 0 \\
& \alpha \geqslant 0 .
\end{aligned}
$$

Various versions of linear programming computer programs using the Simplex 
algorithm of Dantzig (1963) originally proposed in 1947 are available such as IBM supplied MPS 360 program. We shall use the programs given by Cuer and Bayer (1980) who have provided the following innovations (using recent numerical results) in their formulations:

(i) To invert matrices for calculating bases, the Bartels Golub decomposition of matrices (Bartels and Golub 1969; Bartels 1971) is used. The problem due to the addition or deletion of a column can be handled easily. Stability in the computation is increased by scaling the variables.

(ii) Choice of the incoming nonbasic variable to the basic variable set is done by taking the steepest edge (Kuhn and Quandt 1963; Reid 1975; Goldfarb 1976) in searching the vertices of the polyhedron on which the solution could lie.

In the present paper we shall first present the mathematical solution for the basal heat problem without the radiogenic heat source and next consider the problem with the heat source. We then obtain an ideal body solution analytically for a small number of observed surface heat flow values using Parker's formulation for a synthetic example. For larger data sets, the ideal body solution has been obtained using a linear programming method as developed by Cuer and Bayer (1980), again on the synthetic example. The method has been further used on a synthetic example wherein radiogenic heat sources have been included. After showing its usefulness for the synthetic case, a real example of inferring high Moho heat flow in the French Massif Central has been considered. The presence of a high heat flow zone at the Moho depth has been confirmed. We should add that the inverse problem of the estimation of basal temperature variations has already been considered by Huestis $(1980,1981)$ and applied over Sierra Nevada and the Basin and Range provinces. We have taken a different boundary condition, because in certain cases, such as the French Massif Central, it would be more realistic to prescribe heat flux boundary condition rather than temperature boundary condition. Nature of the basal boundary condition on the surface heat flow has recently been discussed by Nakiboglu and Lambeck (1985).

\section{Inverse basal anomalous heat flow problem without radiogenic heat sources}

The problem can be written as one of determining $q_{L}(X)$ at some depth $Z=L$, given the knowledge of the surface heat flow, $q_{s}(X)$. We shall first derive this relationship. The differential equation governing the temperature distribution in the layer $Z \in(0, L)$ is

$$
\left(\partial^{2} / \partial X^{2}\right)+\left(\partial^{2} / \partial Z^{2}\right) T=0
$$

where $X(Z)$ is the horizontal (vertical, positive downward) coordinate. The boundary conditions are

$$
\begin{aligned}
& T=0, \quad \text { at } Z=0, \\
& K \frac{\partial T}{\partial Z}=q_{0}+q_{L}(X) \quad \text { at } Z=L
\end{aligned}
$$

In (8), $q_{L}$ is the uniform heat flow and $K$, the thermal conductivity.

We can substitute

$$
T=q_{0} Z / K+\theta,
$$


in (6) to (8) to get

$$
\begin{aligned}
& \left(\frac{\partial^{2}}{\partial X^{2}}+\frac{\partial^{2}}{\partial Z^{2}}\right) \theta=\dot{0}, \\
& \theta=0 \text { at } Z=0, \\
& K \frac{\partial T}{\partial Z}=q_{L}(X) \text { at } Z=L .
\end{aligned}
$$

Using the Fourier transformation, we obtain the following relationship between enhanced surface heat flow, $q_{s}(x)$, and $q_{L}(x)$ :

$$
Q_{s}(k)=Q_{L}(k) \operatorname{sech}(k L)
$$

where $Q_{s}(k)$ and $Q_{L}(k)$ are the Fourier transforms of $q_{s}(x)$ and $q_{L}(x)$ respectively.

This equation reduces in space domain as

$$
q_{s}(X)=\int_{-\infty}^{\infty} \frac{q_{L}\left(X^{\prime}\right)}{2 L} \operatorname{sech}\left[\pi\left(X-X^{\prime}\right) / 2 L\right] \mathrm{d} X^{\prime}
$$

using the inverse Fourier transform of $\operatorname{sech}(k L)$ as $\operatorname{sech}[\pi x / 2 L]$ (from Campbell and Foster 1957). Let us non-dimensionalise this equation by substituting

$$
\left(X, X^{\prime}\right)=\left(x, x^{\prime}\right) \cdot 2 L
$$

We thus get

$$
q_{s}(x)=\int_{-\infty}^{\infty} q_{L}\left(x^{\prime}\right) \operatorname{sech}\left[\pi\left(x-x^{\prime}\right)\right] \mathrm{d} x^{\prime} .
$$

This is the relationship for deriving $q_{L}(x)$ given $q_{s}(x)$. We shall first calculate Parker's ideal body solution following the formulation by Huestis (1982). Suppose we have two data, one at $x=0$ and another at $x=x_{1}$. We thus have from (16)

$$
\begin{aligned}
& q_{s}(0)=\int_{a}^{b} q_{L}\left(x^{\prime}\right) \operatorname{sech}\left(\pi x^{\prime}\right) \mathrm{d} x^{\prime}, \\
& q_{s}\left(x_{1}\right)=\int_{a}^{b} q_{L}\left(x^{\prime}\right) \operatorname{sech}\left(\pi\left(x_{1}-x^{\prime}\right) \mathrm{d} x^{\prime}\right) .
\end{aligned}
$$

Here we have assumed that

$$
q_{L}(x)=0 \text { for } x \notin(a, b)
$$

Equations (17) and (18) can be recast, by using (so that the range of integration becomes 0 to 1 ):

as

$$
x^{\prime}=(b-a) y+a,
$$

$$
\begin{aligned}
& q_{0}=q_{s}\left(y_{1}\right)=(a-b) \int_{0}^{1} q_{L}(y) \operatorname{sech}[\pi[(b-a) y+a]] \mathrm{d} y \\
& q_{1}=q_{s}\left(y_{2}\right)=(a-b) \int_{0}^{1} q_{L}(y) \operatorname{sech}\left[\pi\left[(b-a)\left(y_{2}-y\right)\right]\right] \mathrm{d} y, \\
& \left(y_{1}=-a /(b-a) ; y_{2}=\left(x_{1}-a\right) /(b-a)\right)
\end{aligned}
$$


Equations (21) and (22) can be further changed so that the integral of data kernels $\left(H_{1}\right.$ and $H_{2}$ in eq. (23) and (24)) be 1 :

$$
\begin{aligned}
& C_{1}=q_{0} / N_{1}=\int_{0}^{1} q_{L}(y) H_{1}(y) \mathrm{d} y, \\
& C_{2}=q_{1} / N_{2}=\int_{0}^{1} q_{L}(y) H_{2}(y) \mathrm{d} y,
\end{aligned}
$$

where

$$
\begin{aligned}
& H_{1}(y)=(b-a) \operatorname{sech}[\pi[(b-a) y+a]] / N_{1} \\
& H_{2}(y)=(b-a) \operatorname{sech}\left[\pi\left[(b-a)\left(y_{2}-y\right)\right]\right] / N_{2} \\
& N_{1}=\left[\tan ^{-1}(\operatorname{sech}(\pi b))-\tan ^{-1}(\sinh (\pi a))\right] / \pi \\
& N_{2}=\left[\tan ^{-1}\left(\operatorname{sech}\left(\pi(b-a) y_{2}\right)\right)-\tan ^{-1}\left(\sinh \left(\pi(b-a)\left(y_{2}-1\right)\right)\right)\right] / \pi
\end{aligned}
$$

If $C_{1}>C_{2}$, the ideal body of Parker (1974) (see Huestis 1982) is specified by

$$
q_{L}(y)=\left\{\begin{array}{l}
q_{0} ; a_{1} H_{1}+a_{2} H_{2}>0 \\
0 ; a_{1} H_{1}+a_{2} H_{2}<0,
\end{array}\right.
$$

where $a_{1}$ and $a_{2}$ are constants and $q_{9}$ is the desired bound which gives a minimum of

$$
\max \left|q_{L}(y)\right| \text {. }
$$

Zero-crossing of $a_{1} H_{1}+a_{2} H_{2}$ within $y \in(0,1)$ occurs at a point, say $y_{0}$. In that case the ideal body can be given as

$$
q_{L}(y)=\left\{\begin{array}{l}
q_{0} ; 0 \leqslant y<y_{0}, \\
0 ; y_{0} \leqslant y \leqslant 1 .
\end{array}\right.
$$

Thus the problem reduces to the determination of $q_{0}$ and $y_{0}$ which gives the strength and the geometry of the 'ideal body' anomalous zone. This can be easily obtained using (23) and (24). We thus get

$$
\begin{aligned}
& q_{0} \int_{0}^{y_{0}} H_{1}(y) \mathrm{d} y=C_{1} \\
& q_{0} \int_{0}^{y_{0}} H_{2}(y) \mathrm{d} y=C_{2}
\end{aligned}
$$

Given the data $C_{1}$ and $C_{2}$, we can find $q_{0}$ and $y_{0}$.

We have performed the above calculations over a synthetic example. We take $a=$ $-b=1$. We further take for $q_{L}\left(\mathrm{mWm}^{-2}\right)$ (see figure 1)

$$
q_{L}=20 \cdot 9[H(x+\cdot 3)-H(x-3)] \text {. }
$$

Suppose we are given two observations at $x=0$ and $x=x_{1}$. We first solve the direct problem to calculate data $C_{1}$ and $C_{2}$. We then use this data to obtain the nature of the ideal body solution. We illustrate the use of these calculations in figure 2 . Here we show the estimation of $q_{0}$ and $y_{0}$ as a function of $x_{1}$ which indicates the separation of the observation points. 


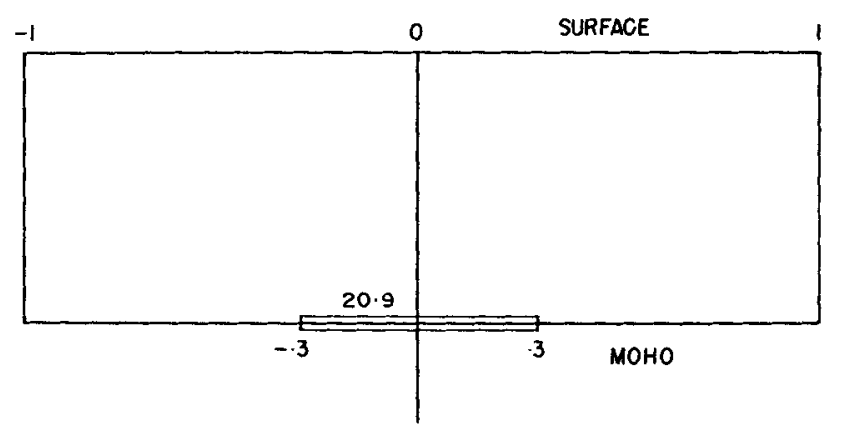

Figure 1. Geometrical configuration of the synthetic crustal model without heat source.

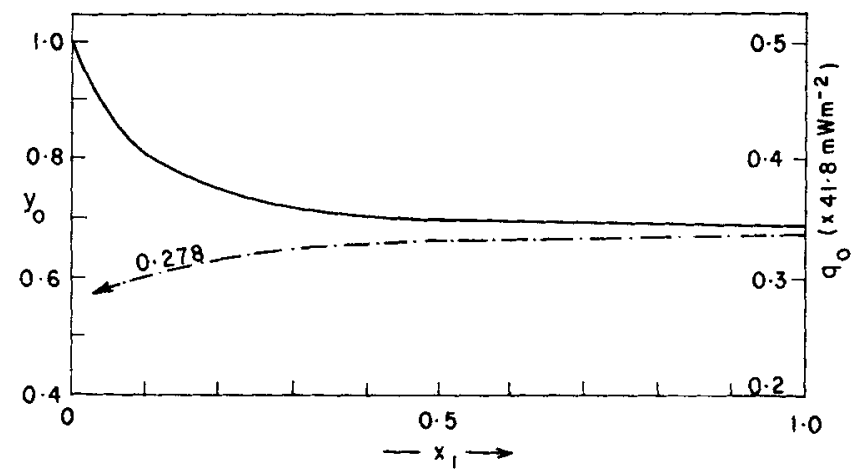

Figure 2. Analytical ideal body solution of the crustal model without heat source.

The curve reveals that for $x_{1}=1$, the ideal body is given by:

$$
\begin{aligned}
& q_{L}(y)=\left\{\begin{array}{l}
14 \cdot 21,0 \leqslant y \leqslant 0.7 \\
0,0 \cdot 7<y \leqslant 1,
\end{array}\right. \\
& q_{L}(y)=\left\{\begin{array}{l}
12 \cdot 25 ; 0 \leqslant y \leqslant 0 \cdot 8, \\
0 ; 0.8<y \leqslant 1 .
\end{array}\right.
\end{aligned}
$$

Thus the size of the ideal body is smaller and the value of the anomaly is higher if both heat flow sites are well separated. Given these two observations, one can assert that the true maximum of heat flow anomaly at depth would exceed these 'ideal body' values.

When more than 2 data are considered, this approach cannot be used. However, ideal body solutions can still be obtained numerically using the approach of Cuer and Bayer (1980).

We take the model as:

$$
q_{L}=41 \cdot 8[H(x+\cdot 3)-H(x-\cdot 3)]
$$

and discretize the domain $x \in(-1,+1)$ into 20 blocks of equal size. Taking successively 2,3 and 5 data we obtain the solution giving the smallest supremum of $q_{L}$ on the whole range. These solutions are plotted in figure 3 , which indicate that with 


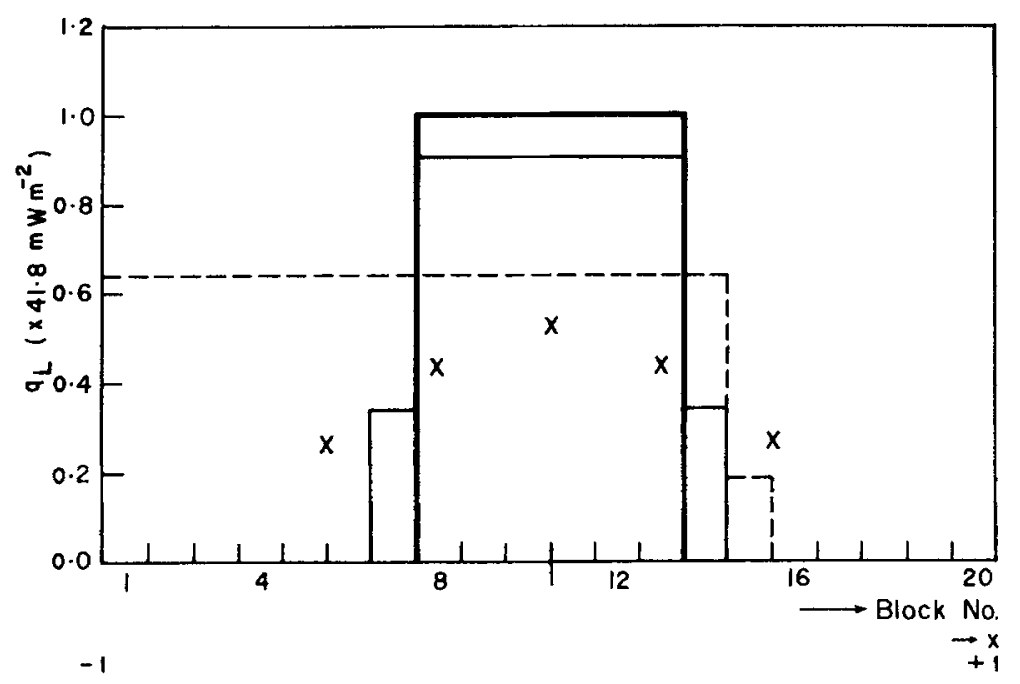

Figure 3. Estimates of the basal heat flux for the crustal model without heat source ( $x$-input heat flow data_— model, -.- using two data points, _ _ using three data points).

a few observations ( 3 or 5 which give the same result) one can resolve the heat flow anomaly quite well.

\section{Inverse basal anomalous heat flow problem with radiogenic heat production}

We have in this case the following mathematical model:

$$
\begin{aligned}
K\left(\frac{\partial^{2}}{\partial X^{2}}+\frac{\partial^{2}}{\partial Z^{2}}\right) T & =-A_{1} \\
T & =0, \quad \text { at } Z=0 \\
K \frac{\partial T}{\partial Z} & =q_{L}(X), \quad \text { at } Z=L \\
K \frac{\partial T}{\partial Z} & =0, \quad \text { at } X=0 \text { and } \\
X & =L_{1} .
\end{aligned}
$$

We have assumed (41) to limit the size of the model. We can non-dimensionalise the problem by assuming

$$
\begin{aligned}
\left(x^{*}, z^{*}\right) & =(X, Z) / L, \\
T & =q_{0} L \theta / K .
\end{aligned}
$$

We then get after dropping*s,

$$
\left(\frac{\partial^{2}}{\partial x^{2}}+\frac{\partial^{2}}{\partial z^{2}}\right) \theta=A
$$




$$
\begin{gathered}
\theta=0 \quad \text { at } z=0 \\
\frac{\partial \theta}{\partial z}=q(x) \quad \text { at } z=1 \\
\frac{\partial \theta}{\partial x}=0 \quad \text { at } x=0 \text { and } 1
\end{gathered}
$$

where

$$
A=A_{1} L / q_{0} \quad q=q_{L}(x) / q_{0}, \quad l=L_{1} / L .
$$

We can use Fourier cosine transformation in $x$. We then get

$$
\begin{aligned}
\theta_{c}= & M \exp (n \pi z / l)+N \exp (-n \pi z / l) \\
& -\frac{l}{n \pi} \int_{0}^{l} A_{c}\left(z^{\prime}, n\right) \sinh \left(\frac{n \pi}{l}\left(z-z^{\prime}\right)\right) \mathrm{d} z^{\prime}
\end{aligned}
$$

where $M$ and $N$ are constants and

$$
\theta_{c}\left[A_{c}\right]=\int_{0}^{1} \theta[A] \operatorname{Cos}\left(\frac{n \pi x}{l}\right) \mathrm{d} x
$$

Applying the boundary conditions, we get the transform of the surface heat flow as

$$
q_{s c}=q_{c} \operatorname{sech}\left(\frac{n \pi}{T}\right)+\int_{0}^{1} A_{c}\left(z^{\prime}, n\right) \operatorname{Cosh}\left[\left(1-z^{\prime}\right) \frac{n \pi}{T}\right] \operatorname{sech}(n \pi / l) \mathrm{d} z^{\prime} .
$$

Using inverse Fourier cosine transformation, we get

$$
\begin{aligned}
q_{s}(x)= & \int_{0}^{l} q\left(x^{\prime}\right) M\left(x, x^{\prime}\right) \mathrm{d} x^{\prime} \\
& +\int_{0}^{1} \mathrm{~d} z^{\prime} \int_{0}^{1} \mathrm{~d} x^{\prime} A\left(x^{\prime}, z^{\prime}\right) \cdot N\left(x, x^{\prime}, z^{\prime}\right) .
\end{aligned}
$$

where

$$
\begin{aligned}
M & =\left(\frac{1}{l} \delta_{n 0}+\frac{2}{l} \sum_{n=1}^{\infty} \frac{\operatorname{Cos}(n \pi x / l)}{\operatorname{Cosh}(n \pi / l)} \operatorname{Cos}\left(n \pi x^{\prime} / l\right)\right) \\
N & =\left[\frac{1}{l} \delta_{n 0}+\frac{2}{l} \sum_{n=1}^{\infty} \frac{\operatorname{Cos}(n \pi x / l)}{\operatorname{Cosh}(n \pi / l)} \operatorname{Cosh}[(1-z) n \pi / l] \times \operatorname{Cos}\left(n \pi x^{\prime} / l\right)\right] .
\end{aligned}
$$

Now we can pose the inverse problem as one of determining $q(x)$ and $A(x, z)$ knowing the values of $q_{s}$. We shall use the approach of Safon et al (1977) and Cuer and Bayer (1980).

We discretize the problem by approximating $q$ and $A$ by piecewise constant functions. In that case we have to obtain the following integrals:

$$
\bar{I}_{1}=\int_{x_{i}}^{x_{i+1}} q\left(x^{\prime}\right) M\left(x, x^{\prime}\right) \mathrm{d} x^{\prime}
$$

and

$$
\bar{I}_{2}=\int_{x_{1}}^{x_{i+1}} \int_{z_{i}}^{z_{i+1}} \mathrm{~d} z^{\prime} A\left(x^{\prime}, z^{\prime}\right) N\left(x, x^{\prime}, z^{\prime}\right)
$$


We can easily evaluate it. We thus get

$$
\begin{aligned}
\bar{I}_{j i}= & \frac{l q_{i}}{n \pi}\left[\frac{1}{l} \delta_{n 0}+\frac{2}{l} \sum_{n=1}^{\infty} \frac{\operatorname{Cos}\left(n \pi x_{j} / l\right)}{\operatorname{Cosh}(n \pi / l)}\right]\left[\sin \left(n \pi x_{i+1} / l\right)-\sin \left(n \pi x_{i} / l\right)\right] \\
\bar{I}_{j i}= & A_{i}\left(\frac{l}{n \pi}\right)^{2}\left[\frac{1}{l} \delta_{n 0}+\frac{2}{l} \sum_{n=1}^{\infty} \frac{\operatorname{Cos}\left(n \pi x_{j} / l\right)}{\operatorname{Cosh}(n \pi / l)}\right] \\
& \times\left[\sin \left(n \pi x_{i+1} / l\right)-\sin \left(n \pi x_{i} / l\right)\right] \cdot\left[\sinh \left(n \pi\left(1-z_{i}\right) / l\right)\right. \\
& \left.-\sinh \frac{n \pi\left(1-z_{i+1}\right)}{l}\right] .
\end{aligned}
$$

Here we have taken

$$
\begin{aligned}
& q_{i}=q(x)\left[H\left(x-x_{i}\right)-H\left(x-x_{i+1}\right)\right], \\
& A_{i}=A(x, z)\left[H\left(x-x_{i}\right)-H\left(x-x_{i+1}\right)\right] .
\end{aligned}
$$

We thus have a discrete diversion of (52) as:

$$
\begin{array}{ll}
q_{s j}=\bar{I}_{j i} q_{i}+\overline{\bar{I}}_{j m} A_{m}, & j=1, \ldots r, \\
i & =1, \ldots p, \\
& m=1, \ldots s . \\
I \mathbf{d}=\mathbf{q}_{s}, &
\end{array}
$$

where

$$
I=[\bar{I}, \overline{\bar{I}}] \text { and } \mathbf{d}=[\mathbf{q}, \mathbf{A}]^{\mathrm{T}} \text {. }
$$

The system of (62) for estimating $d$ is under-determined. One way to proceed further is to fix bounds on various variables and to look at the external solution for every unknown, $q$. This can be achieved by the use of the linear programming technique. In this direction, an interesting approach is the ideal body solution and we would again use the numerical scheme of Cuer and Bayer (1980) for this determination. We shall first use it over a synthetic example and then take up a real case.

\subsection{Synthetic example}

The model used is shown in figure 4; it consists of a $100 \mathrm{~km} \times 30 \mathrm{~km}$ domain. The surface heat flow results from a layered heat production and of a bottom heat flow of $30 \mathrm{mWm}^{-2}$ for $X \geqslant 70 \mathrm{~km}$ and $X \leqslant 30 \mathrm{~km}$ and of $60 \mathrm{mWm}^{-2}$ for $30<X<70 \mathrm{~km}$. Five heat flow data with an error at $\pm 2 \mathrm{mWm}^{-2}$ are given at $X=0,25,50,75,100 \mathrm{~km}$. The discretization of the problem involves 50 prismatic blocks for the heat production and 10 segments $(10 \mathrm{~km}$ long) for the bottom heat flow. In each block the heat production is assumed to be known within some bounds which will be discussed later. $A$ priori bounds at 30 to $100 \mathrm{mWm}^{-2}$ for $q_{L}$ are chosen.

Let us consider the case where the heat production model is very well defined (i.e. bounds at $\pm 0.05 \mu \mathrm{Wm}^{-3}$ ). Taking these bounds into account we computed the minimum and maximum values at $q_{L}$ in each segment using the Cuer and Bayer (1980) procedure. These $\min / \max$ can be displayed as a range where all compatible solutions 


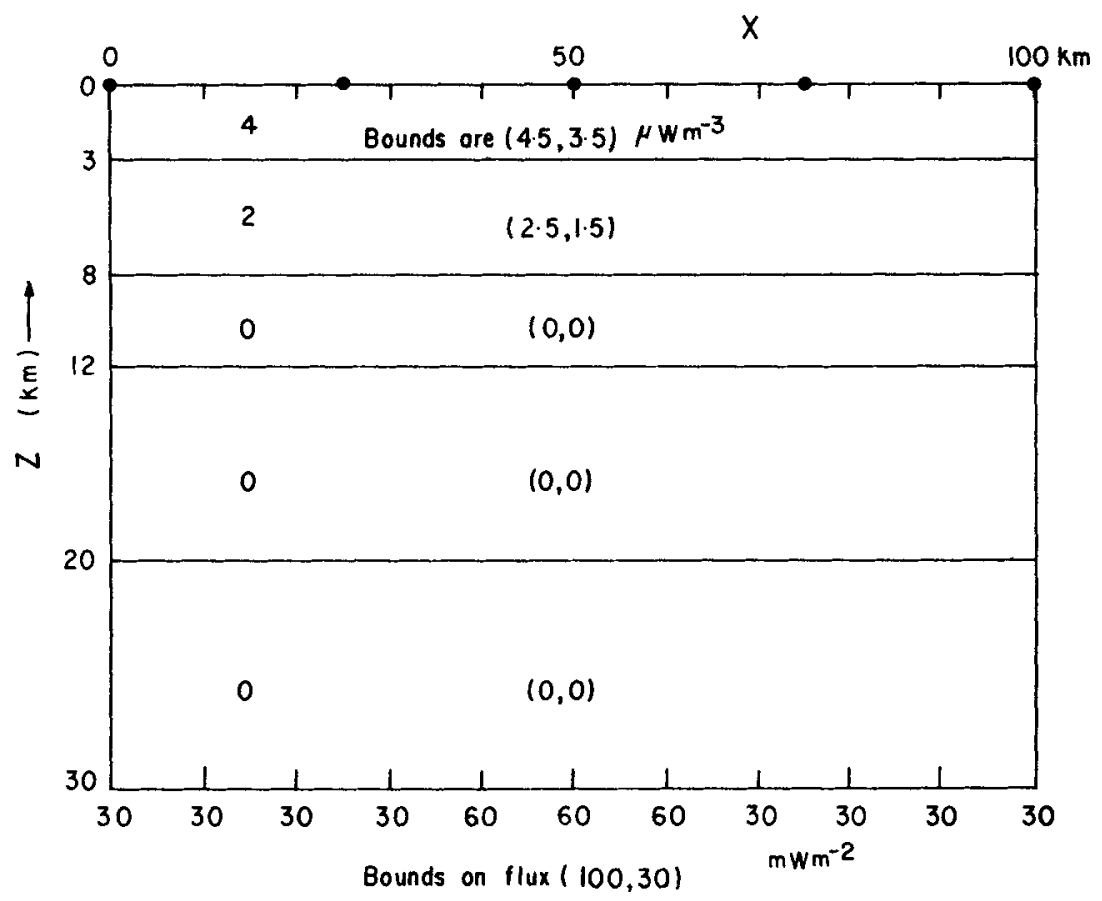

Figure 4. Geometrical configuration of the synthetic crustal model with heat source.

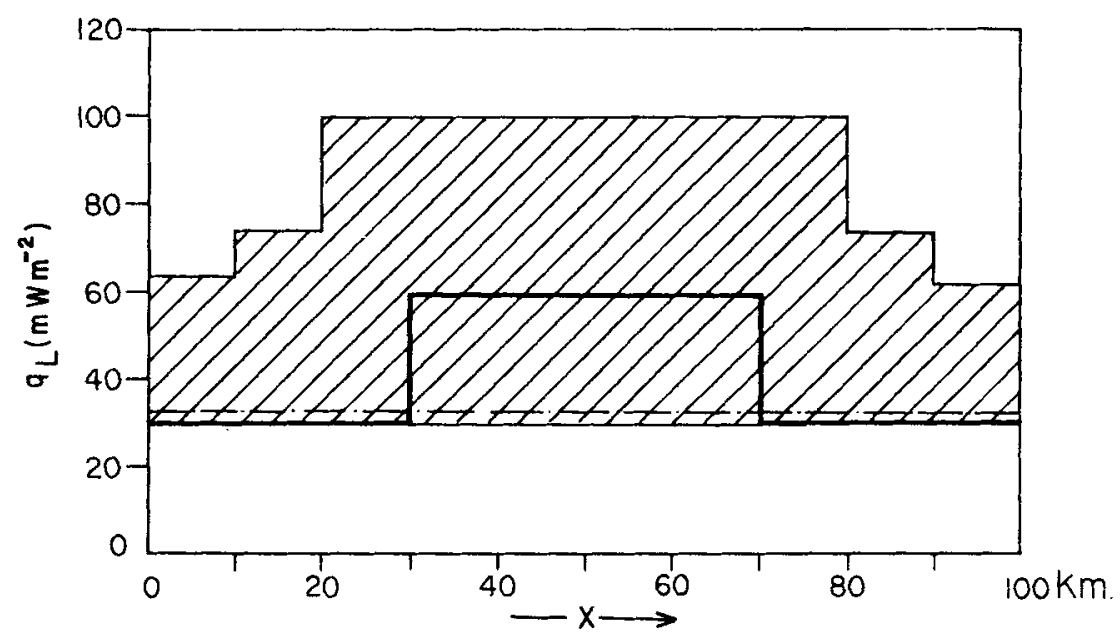

Figure 5. Estimates of the basal heat flux from a synthetic model (---ideal body, —-model, the hatched area covers the range of possible solutions).

of the problem lie. This is shown in figure 5. The range of possible solutions is very wide; however the maximum of the envelope reproduces a maximum similar to the actual variation of $q_{L}$. The ideal body solution corresponds to a constant value of $30.9 \mathrm{mWm}^{-2}$ i.e. close to the assumed lower bound. It is therefore, clear that uncertainities in heat production result in a degradation of the resolution of the basal heat flow. 


\subsection{Real case study}

The proposed technique can now be applied to a real case where the presence of a mantle heat flow anomaly has already been proposed. The area under study is the French Massif Central which is outlined by a positive heat flow anomaly of broad wavelength (Vasseur and Lucazeau 1982). This heat flow anomaly was interpreted as partly due to the presence of rocks with enhanced radioactivity and also to the existence of a mantle heat flow anomaly related to the Cenozoic volcanism, whose manifestations have been recognized (Lucazeau and Vasseur 1981). In order to evaluate the crustal contribution to the surface heat flow many geochemical and geophysical data were used in order to propose values of heat production in various segments of the crust. The basic data are then 5 heat flow data on an $\mathrm{E}$. W. profile, estimates of heat production in the various layer of the crust.

The a priori information for heat production is synthesized in table 1 giving a vertical cross section of the earth crust along the E-W direction. The structure of the earth crust is deduced from deep seismic soundings (Perrier and Ruegg 1977) and numerous heat production measurements have been obtained on various geologic units (Lucazeau and Vasseur 1981). Between 0 and $32 \mathrm{~km}$ depth the crust is divided into 210 rectangular blocks (figure 6); in each block an upper and a lower bound are set, taking into account the various uncertainities on the heat production measurements and on the geological structure. For the unknown bottom heat flow $q_{L}$, the horizontal distance is discretized into 5 steps; a priori bounds of 20 to $100 \mathrm{mWm}^{-2}$ are assumed for each unknown (figure 6).

Using a Linear programming approach we can compute various solutions of the system, i.e. models of $q_{L}$ and $A$ which are consistent with the data and with the a priori

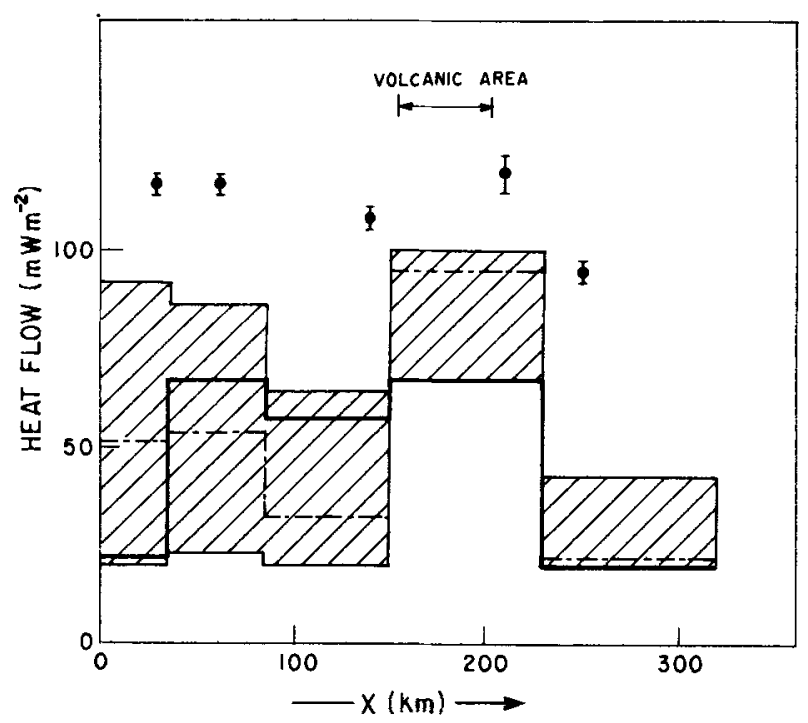

Figure 6. As a function of horizontal distance are plotted: the surface heat flow data with error bars as dots (in $\mathrm{mWm}^{-2}$ ); the range of possible solutions for the bottom heat flow (hatched area); the solution which gives $\min \operatorname{Sup}\left|q_{L i}\right|$ as heavy line; the solution which gives $\min \operatorname{Sup}\left|A_{i}-A_{0 i}\right|$ as broken line. 
Table 1. East-west vertical cross section of the earth's crust. This section is divided into rectangular blocks (irregular in size due to surface geology). In each block the upper and lower

W figures give the upper and lower bounds of heat production (in $\mu \mathrm{Wm}^{-3}$ ).

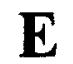

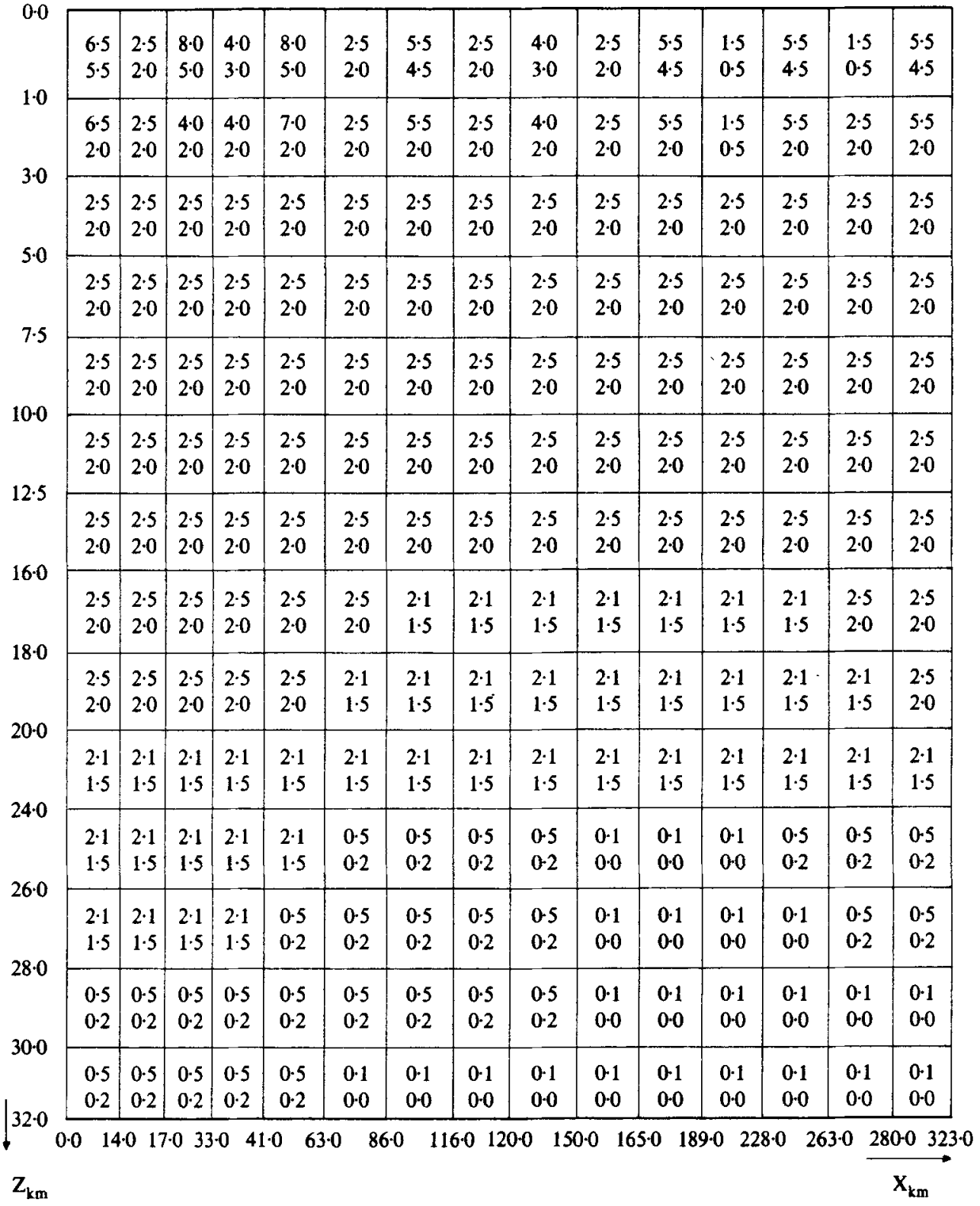

bounds. Various solutions are presented in figure 6; only those components of the solution corresponding to the bottom heat flow are displayed.

First we obtain solutions giving maximum and minimum values of $q_{L}$ in each step. The corresponding range of solutions (hatched area) is quite large, mainly in the 
western part; however under the "volcanic area" (figure 6), the minimum $q_{L}$ is clearly enhanced.

Two particular solutions are also shown in figure 6: the solution which gives the smallest supremum of $q_{L}$ (ideal body on $q_{L}$ ) and the solution which gives the minimum of:

$$
\operatorname{Sup}\left|A_{i}-A_{0 i}\right|
$$

where $A_{0 i}$ is the average estimated heat production in each block (table 1). The first solution corresponds to the "ideal body" for $q_{L}$ : it yields a smallest supremum of $67 \mathrm{mWm}^{-2}$.

These results compare well with the model presented by Lucazeau and Vasseur (1981) and the presence of a strong perturbation of bottom heat flow around the volcanic area seems to be ascertained. However, in the western part the existing data cannot bring out any firm conclusion for the origin of high heat flow in this part of the studied area.

\section{Acknowledgements}

This work was started while RNS was on visit to CGG (CNRS), France. Authors are grateful to authorities in CSIR and CNRS and the Directors of NGRI and CGG for making this joint collaboration possible.

Discussion with Drs R Bayer and M Cuer have been most helpful and we are grateful to them.

\section{References}

Backus G E 1972 Mathematical problems in the geophysical sciences vol. 2 (ed.) W H Reid (Providence: Am. Math. Soc.) p. 1

Backus G E and Gilbert F 1967 Geophys. J. R. Astron. Soc. 13247

Backus G E and Gilbert F 1968 Geophys. J. R. Astron. Soc. 16169

Backus G E and Gilbert F 1970 Philos. Trans. R. Soc. Lond. 226123

Bartels R H 1971 Numer. Math. 16414

Bartels R H and Golub G H 1969 Commun, ACM 12206

Bayer R and Cuer M 1981 Ann. Geophys. 37347

Bayer R and Lesquer A 1978 Bull. Soc. Geol. Fr. 20863

Blackwell D D, Bowen R G, Hull D A, Riccio J and Steele J L 1982 J. Geophys. Res. 878735

Campbell G A and Foster R M 1957 Fourier integrals for practical applications (Princeton: D Von Nostrand) p. 177

Cuer M and Bayer R 1980 Geophysics 451706

Dantzig G B 1963 Linear programming and extensions (New York: Princeton University Press)

Francheteau J, Jaupart C, Jie S X, Wen-Hua K, De-Lu L, Jia-Chi B, Hung-Pin W and Hsia-Yeu D 1984 Nature (London) 30732

Garmany J, Orcutt J A and Parker R L 1979 J. Geophys. Res. 843615

Goldfarb D 1976 Sparse matrix computations (eds) J R Bunch and D J Rose (New York: Academic Press)

Grasso J R, Cuer M and Pascal G 1983 Geophys. J. R. Astron. Soc. 75437

Huestis S P 1979 Geophys. J. R. Astron. Soc. 58249

Huestis S P 1980 Geophys. J. R. Astron. Soc. 62649

Huestis S P 1981 Geophys. J. R. Astron. Soc. 65165

Huestis S P 1982 Geophys. J. R. Astron. Soc. 69253

Huestis S P and Parker R L 1977 J. Geophys. Res. 825293 
Jackson D D and Matsu'ura M 1985 J. Geophys. Res., 90581

Kuhn H W and Quandt R E 1963 Proc. 15th Symp. Appl. Maths. (Providence: Am. Math. Soc.) p. 107 Lucazeau F and Vasseur G 1981 Ann. Geophys. 3789

Nakiboglu S M and Lambeck K 1985 J. Geophys. Res. 902985

Oldenburg D N 1983 J. Geophys. Res. 887387

Parker R L 1974 Geophysics 39644

Parker R L 1975 Geophys. J. R. Astron. Soc. 42315

Parker R L 1977a Annu. Rev. Earth Planet. Sci. 535

Parker R L 1977b Rev. Geophys. Space Phys. 15446

Perrier G and Ruegg J C 1973 Ann. Geophys. 29435

Reid J K 1975 Lecture notes in mathematics. No. 506. Springer-Verlag, p. 176

Richards V, Bayer R and Cuer M 1984 Geophysics 491781

Sabatier P C 1977a J. R. Astron. Soc. 48443

Sabatier P C 1977b Geophys. J. R. Astron. Soc. 48415

Safon C, Vasseur G and Cuer M 1977 Geophysics 421215

Sclater J G, Jaupart C and Galson D 1980 Rev. Geophys. Space Phys. 18269

Spensor C 1985 Geophys. J. R. Astron. Soc. 80619

Tarantola A and Valette B 1982 Rev. Geophys. Space Phys. 20219

Vasseur G and Lucazeau F 1982 Geothermics and geothermal energy (eds) V Cermak and R Haenel (Schwieizerbart: Sche Verlagsbuch handlung) p. 79 